\title{
Thermo-Mechanical Properties of ABS/CB Polymer Nanocomposites
}

\author{
Alok Kumar Pandey ${ }^{1}$ and Kamal K. Kar ${ }^{1,2^{*}}$ \\ Advanced Nanoengineering Materials Laboratory \\ ${ }^{1}$ Materials Science Programme and ${ }^{2}$ Department of Mechanical Engineering, Indian Institute of \\ Technology Kanpur, Kanpur-208016, India \\ *Email: kamalkk@iitk.ac.in
}

Polymer nanocomposites (NCs) consisting of polymer as matrix and filler material as reinforcement have found applications in many areas of science and technology. In practice, the incorporation of carbon black (CB) in the polymer do not necessarily improves the properties of the NCs dramatically. With an increase in the filler concentration, the chances of agglomeration increases and also affects the thermo-mechanical characteristics of the NCs. Acrylonitrile butadiene styrene (ABS) has been widely used as a packaging material to cover electronic items and electrical enclosures but the issues of concern are mechanical strength and the problem of heat dissipation [1-2]. In order to overcome these issues, $\mathrm{CB}$ has been reinforced into ABS matrix in the recent past, due to its unique properties such as, good thermal and electrical conductivities [3]. In this study, ABS/CB NCs have been prepared by vertical twin-screw micro compounder and microinjection molding. The concentration (in vol\%) of $\mathrm{CB}$ is varied from 0 to 40 in the ABS/CB NCs.

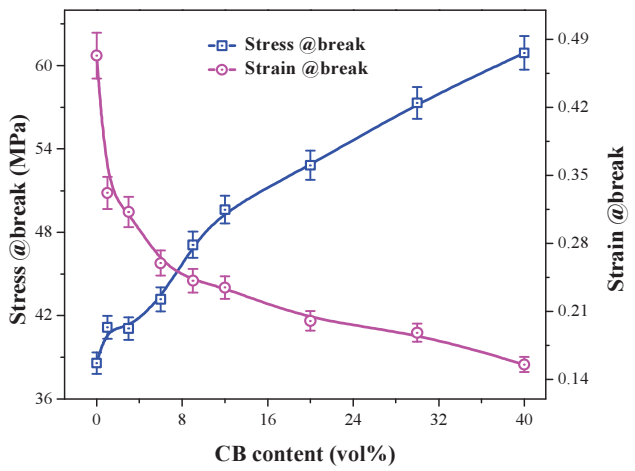

Figure 1: Stress and strain at breaking point for $\mathrm{ABS} / \mathrm{CB} \mathrm{NCs}$ at varying $\mathrm{CB}$ concentration

The thermal, mechanical and thermomechanical properties of the NCs have been studied. An increase in the ultimate tensile strength from 49.3 to $60.2 \mathrm{MPa}$ is observed with a reduction in the percentage elongation.
Stress at break increases by $57 \%$ while the strain at break first decreases sharply till $6 \mathrm{vol} \%$ $\mathrm{CB}$ addition and finally settle down with $67 \%$ decrease for NCs having $40 \mathrm{vol} \% \mathrm{CB}$ content, as shown in Figure 1. The observed melt viscosity during processing of $\mathrm{NCs}$ has been found to increase by $58 \%$ with an increasing $\mathrm{CB}$ concentration, as shown in Figure 2.

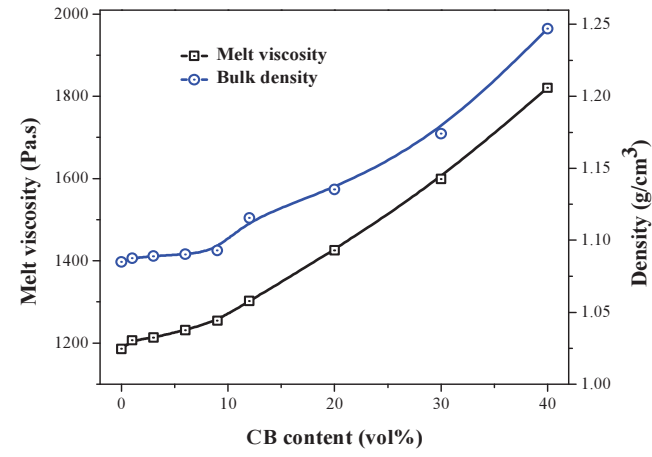

Figure 2: Variation in melt viscosity and bulk density for varying CB content

Bulk density measurement exhibits slight amount of increase till 9 vol\% CB addition, further increase in the $\mathrm{CB}$ concentration increases the bulk density of NCs linearly (Figure 2). A significant improvement in the storage and loss modulus (measured via dynamic mechanical thermal analysis) as well as in the thermal conductivity has been observed with respect to an increase in the $\mathrm{CB}$ concentration. This study shows that the ABS/CB NCs show improved properties when compared to that of the pristine ABS.

\section{References}

1. K. K. Kar, A. Hodzic, Development in Nanocomposite. Singapore, Research Publishing Services, 2014.

2. K. K. Kar, S. Srivastava, A. Rahaman, S. K. Nayak, Polm. Compos. 29 (2008) 489.

3. A. Shenavar, F. Abbasi, J. Appl. Polym. Sci. 105 (2007) 2236. 